\title{
Applying the CHECk tool to Participatory Design Sessions with Children
}

\author{
Maarten Van Mechelen \\ Bieke Zaman \\ Karin Slegers \\ CUO | Social Spaces, iMinds, KU Leuven \\ Parkstraat 45/3605, 3000 Leuven, Belgium \\ maarten.vanmechelen@soc.kuleuven.be \\ bieke.zaman@soc.kuleuven.be \\ karin.slegers@soc.kuleuven.be
}

\author{
Gavin Sim \\ Peggy Gregory \\ Matthew Horton \\ ChiCl Group, UCLan \\ Preston, PR1 2HE, UK \\ GRSim@uclan.ac.uk \\ AJGregory@uclan.ac.uk \\ MPLHorton@uclan.ac.uk
}

\begin{abstract}
To encourage ethical practices in participatory design with children the CHECk tool was created. This paper reports on an expert review of the CHECk tool and a validating case study. Four main challenges to the CHECk tool are identified: (1) how to inform children on the research and their role herein, (2) distinguishing between project values and designer or researcher's personal values, (3) accounting for the dynamic nature and social constructedness of values in design, and (4) the emergence of values in all stakeholders including child design partners. We advocate complementing CHECk with interactive storytelling and show how this narrative can be used to not only inform participation and achieve ethical symmetry, but also to negotiate values with child design partners.
\end{abstract}

\section{Categories and Subject Descriptors}

K 4.1 [Public Policy Issues] ethics

\section{General Terms}

Human Factors

\section{Keywords}

CCI; Participatory Design; Value Sensitive Design; ethics

\section{INTRODUCTION}

Within the area of Child Computer Interaction (CCI) children have participated in the design of technology for over two decades using a variety of established methods, e.g. [1][6].

Technology has moral impacts on users and their environment, it shapes their lives and practices in important ways. Technology is therefore not merely enabling but constitutive. On the other hand, users may appropriate technology for purposes other than those intended in design and, by doing so, technology's functionality is adjusted and changed. Such an interactional position holds that values are not solely designed into technology, nor are they solely conveyed by social drivers and forces, it works both ways [12].

Permission to make digital or hard copies of all or part of this work for personal or classroom use is granted without fee provided that copies are not made or distributed for profit or commercial advantage and that copies bear this notice and the full citation on the first page. Copyrights for components of this work owned by others than ACM must be honored. Abstracting with credit is permitted. To copy otherwise, or republish, to post on servers or to redistribute to lists, requires prior specific permission and/or a fee. Request permissions from Permissions@acm.org.

IDC'14, June 17-20, 2014, Aarhus, Denmark.

Copyright 2014 ACM 978-1-4503-2272-0/14/06 ..\$15.00.

http://dx.doi.org/10.1145/2593968.2610465
Since technology should no longer be considered value-neutral, an increasing body of HCI (and CCI) research has concerned itself with understanding how to explore values more explicitly during design and evaluation. At the same time, a number of approaches for systematically considering human values in information technology have emerged, in particular value sensitive design [7][3]. Furthermore, in related fields such as participatory design attempts have been made to rekindle values in what is called a more authentic approach towards participatory design [10]. In this paper we rely on Rokeach's [15] notion of values as something that a person or a group of persons consider(s) important in life, as have many others, e.g. [7][10].

To encourage ethical practice in participatory design with children a value checklist referred to as CHECk was created for use prior to and at the start of design activites [13]. CHECk, consists of two checklists, CHECk 1 and 2, designed to help CCI researchers to critically consider their values when involving children in design projects, and to examine how best to explain participatory design activities to children to aid informed consent [13].

In this paper, we advocate complementing CHECk with interactive storytelling and using this narrative to not only better inform participation and achieve ethical symmetry, but also to negotiate values concerning the project and its outcomes with our child design partners. This way, CHECk becomes a vehicle to open up dialogue and to establish a shared narrative space, that is, a common ground where adults and children can meet.

\section{EXAMINING VALUES AND PARTICIPATION}

Examining your own values as a researcher or designer prior to any design activity is a condition sine qua non to better inform child design partners. Using tools like CHECk fits in a broader general trend in the HCI and CCI community that has often been referred to as the third wave of HCI research [3]. This transition came with a turn to design and culture as new theoretical concerns, indicating a trend towards more critical reflection [11]. It has called for accountability in the ethnomethodological sense in that researchers and designers are increasingly expected to explicitly account for what they are examining, designing and the procedures followed to perform these practices [8]. It does not only call for a responsibility to account for the values that are being designed for, but also for a reflexivity regarding the fact that interaction designers and researchers themselves bring values to the design process [15]. Design and research teams therefore need adequate codes or tools.

However, ethical questions that arise when involving children as design partners are not always considered in a standard ethics 
review (e.g. ownership of ideas). In addition, informed consent documents usually target parents rather than informing both adults and children. Finally, there is an urge for an added layer of ethical discussion, indicating personal responsibility to do more than just the minimum.

In order to support CCI researchers and designers to become accountable for the values that they design for, Read et al. have developed CHECk, a tool to encourage reflexivity, consisting of two checklists [13]. The first checklist, CHECk 1, focuses on examining values by asking six questions to be answered prior to any design activity. The questions challenge the designer or researcher to consider the appropriateness of both the technical solution and the involvement of children. The aim is to become more explicit about the values that drive the work, pushing designers and researchers to the extremes of honesty.

The second checklist, CHECk 2, aims to examine the value of participation to the child design partners. Child design partners should be informed about what they will be doing during the design activities, how their contributions will be disseminated and, although difficult, who has credit for the ideas they come up with during these design activities. The main goal of CHECk 2 is achieving ethical symmetry, that is, full consent from the children instead of only consent by adults [5]. By answering the questions, designers and researchers can make sure children can understand their research.

In this paper, we will investigate CHECk's effectiveness to facilitate critical reflection about ethical issues in CCI and to achieve ethical symmetry in participatory design sessions with children. Furthermore, by complementing CHECk with interactive storytelling and creating a shared narrative space were adults and children can meet, we will explore how the tool can be used as a starting point not only to inform but also to negotiate values with child design partners.

\section{CASE STUDY}

Our study consisted of two parts. Firstly, the first author of this paper performed an expert critique of the CHECk tool. Reflections were discussed in follow-up iterations with the coauthors. Based on the results of the expert review, an extended CHECk tool was suggested. Secondly, the extended CHECk tool was empirically evaluated in a concrete case study that dealt with the design of tangible, digital tools to foster pro-social behavior off- and online within a class group. More particularly, the goal was to strengthen social cohesion and prevent (cyber)bullying. Data were gathered in co-design sessions that took place in two schools in Flanders, Belgium, with a group of 49 children aged 9 to 10 .

\subsection{Reviewing the CHECk Tool}

The results of the expert review on CHECk identified both strengths and opportunities for improvements. Although CHECk was judged to be a useful tool for examining values and participation prior to and at the start of design activities with children, we also identified four challenges that can be tackled to exploit the full potential of the tool.

Firstly, CHECk focuses on 'what' to tell child design partners but no explanation is given on 'how' to best tell it. This is an important issue since the CHECk tool aims to facilitate a better understanding by children about what the project is about and how they will be involved and contribute to it. Therefore, one should carefully consider in what form to bring the information to the children.

Secondly, designers and researchers involved in a project do not necessarily have a 'shared' point of view. A distinction should be made between values that are explicitly supported and adopted in a given investigation or project and designer or researcher's personal values (cf. self-disclosure) [3]. Designers and researchers also bring values to the design process through 'seeing as' and through making design judgment [10]. These personal values do not necessarily correspond with the more general project values.

Thirdly, designers and researchers do not necessarily have a 'fixed' point of view about the project. Their values may be dynamic, they can change as part of the design process due to interactions with other stakeholders as well as the technology being developed, and several viewpoints may co-exist depending on the context [9][10][12]. The use of groups in participatory design furthermore reflects a theoretical commitment to the notion that meanings are socially and collectively produced [4]. Therefore, CHECk, as an ethical probe, should account for changes in values about the project and the technology being developed.

Lastly, CHECk does not fully account for children's values. Only researchers and designers are prompted to examine their values prior to any design activities. Child design partners on the other hand are not given the opportunity to express their values on participation or to negotiate their views on ethical questions such as ownership of ideas. We suggest that, in order to develop ethical practice in participatory design with children, dialogue is required, not only between researchers but also between researchers and child design partners

We argue that interactive storytelling may offer interesting opportunities for the challenges listed above and in particular for how to bring the information to the children and how to account for children's values. Building a story around the design challenge and making the project more tangible by adding persona like characters and a realistic plot may be useful for increasing involvement and helping children better understand the value of participation. Establishing a shared narrative space between adults, as outsiders to children's life-world, and children, creates a common ground to meet on [1][6]. When telling the story, children should be prompted to reflect on the design challenge, the values at stake and the consequences of participation. This way, the narrative becomes a stepping stone to open up dialogue with child design partners. Giving a voice to children who are typically not consulted in research practices and ethical considerations may destabilize existing power structures [17].

In sum, the expert review has revealed four areas for improvements to extend CHECk 1 and 2: (1) considering how to inform children on the research and their role herein, (2) distinguishing between project values and designer or researcher's personal values, (3) accounting for the dynamic nature and social constructedness of values in design, and (4) the emergence of values in all stakeholders including child design partners. We advocate complementing CHECk with interactive storytelling and using this narrative to not only inform participation and achieve ethical symmetry, but also to negotiate values with our child design partners [5].

\subsection{Answering the CHECk Questions}

In accordance to the CHECk protocol [13], we answered the questions of the two checklists.

\subsubsection{CHECK 1 questions}

1. What are we aiming to design?

Tangible, digital tools to stimulate pro-social behavior, off- and online, within a class context to prevent (cyber)bullying from happening in the first place.

2. Why this product? 
Excuse: Bullying behavior, off- and online, is still a widespread problem often related to existing social contexts such as the class. Since (cyber)bullying is a group process in which bystanders play an unmistakable role, we target the whole class as a particular social group.

Honest: We had to choose a target group and a societal problem within an ongoing project. Since preventing and coping with (cyber)bullying is a hot topic in Flanders and abroad, we saw interesting academic opportunities.

\section{What platform or technologies are we planning to use?}

Not yet decided, but our aim is to develop tangible, digital tools that can be used in and around the classroom throughout the year.

\section{Why this platform or technology?}

Excuse: tangible interaction offers interesting opportunities to bridge the gap between the off- and online world of children, and to stimulate pro-social behavior on both levels. Furthermore, tangible, digital tools can easily be embedded in a classroom for structural use throughout the year.

Honest: we wanted to do something with tangible interaction, since it offers more possibilities from a technological innovation point of view compared with a mobile application.

5. Which children will we design with?

Fourth graders (i.e. 9- to 10-year-olds) living in Flanders Belgium.

\section{Why these children?}

Excuse: According to literature, 9- to 10-year-olds are an interesting target group for prevention due to the growing influence of peers and the early uptake of social media.

Honest: One of the researchers involved in the project was looking for an additional case for his $\mathrm{PhD}$ research. Therefore, we chose the exact same target group.

\subsubsection{CHECk 2 questions}

1.1 Why are we doing this project (i.e., summary of CHECK 1)?

By designing tangible, digital tools to foster pro-social behavior off- and online within a class group, we hope to prevent (cyber)bullying. The societal relevance of the problem provides interesting academic opportunities. Also, tangible interaction is an interesting topic from a technological innovation point of view. Finally, 9- to 10-year-olds are an interesting age group for prevention and this target group could also be aligned with an ongoing $\mathrm{PhD}$ research.

\subsection{What do we tell the children?}

We are looking for ways to enhance the class atmosphere and to make sure everybody gets along. We therefore aim to build some kind of technology that you, the children, can use in and around the class throughout the year.

\subsection{Who is funding the project?}

IWT, the Agency for Innovation by Science and Technology in Flanders, Belgium.

\subsection{What do we tell the children?}

We are researchers working at the University of Leuven, this means the university pays us to do research.

\subsection{What might happen in the long term?}

By means of multi-modality analysis we will analyze and interpret the results (i.e., artifacts and explanations). The results will be taken forward to fuel the design process and complement the viewpoints of adults. The final design may be implemented in different schools and released in the market.

\subsection{What do we tell the children?}

Some of your ideas may actually be used but most likely not just one idea but a mix of different ideas from you, the children, as well as ideas from parents, teachers, etc. With all these ideas in mind, we will invent something that we might sell to schools throughout Belgium.

4.1. What might we publish?

Reflections on methodology and results of the participatory design activities with children.

\subsection{What do we tell the children?}

We will write about the activities we will be doing together and the ideas you come up with during these activities. These writings will be published in specialist magazines.

\subsection{Extending CHECk with Storyline}

In the case study, we aimed to evaluate the suggestions for improving the CHECk tool that followed from the expert evaluation. To realize this, we extended CHECk with an interactive storyline. As a starting point for our narrative, we used the results of CHECk 2. The first part of the narrative was about us, about what it means to be a design researcher. The second part contextualized the design challenge by telling a partly fictional story about a schoolteacher, Miss Anneleen. The 23 9-10-year-old boys and girls in her class are having a difficult time. The children don't get along very well and the atmosphere in the class is below zero. A lot of detailed examples were included in the narrative, such as:

"Some children always play together during breaks, while excluding others who would really like to join them."

The teacher tried many things to change the atmosphere for the better, but without success. Therefore, she contacted her brother, a researcher at the university, and asked him for help. Since the brother did not know what to do either, he decided to ask children in other schools to help him solve the problem of the bad atmosphere. Together with these children and a colleague he wants to invent something 'magical' to be used in class to enhance the atmosphere and the team spirit. With this story, our aim was to establish a common ground to meet on and to provide a clear end-goal.

\subsection{Negotiating Values}

The narrative became the leitmotif of the design activities with children. In total 4 design sessions were arranged with the children over a number of weeks. During this first meeting, we also gave the children a sensitizing package [16] with four assignments. Unfortunately there is no room to elaborate on each of the assignments, but in one of the assignments we asked them to draw a class with a bad atmosphere, a class they definitely don't want to be part of. This was an individual assignment carried out at home. The results of this were then used to inform the narrative within the second design session. This way, the design challenge became much more tangible and tailored to children's life-world, creating feelings of ownership and a better understanding of the problem.

When we met the children for the first time in their classroom, we did not tell the story in a one-way fashion. Rather, we combined it with an interactive introduction about us, being researchers and what that means. We asked the children what they think it is that researchers do and why. Next, we introduced the problem of Miss Anneleen and we asked the children about their opinions. We then revealed the purpose of our visit, being asked by Miss Anneleen to help her solving the problem of the bad atmosphere in her class and that we needed their help. Some children were a bit skeptical 
in the beginning and wondered if the story was real. However, because of the many details and the story's realism, the children got excited right away.

Next, we used the answers of CHECk 2 to negotiate and inform participation. We asked questions such as "What should we do if our ideas actually solve the problems in Anneleen's class?" and "What if we earn money with an invention based on our ideas?" Formulating these and other questions, we tend to use 'our' and not 'your' ideas, since the designer or researcher facilitating the activities will actively contribute as well. These questions evoked interesting debates, for example between a boy wanting to buy a PlayStation for class use and a girl proposing to use the money to help children in other schools.

"I think, that uh, we should use the money to help children in other schools as well, and so, that the class atmosphere can improve their as well, in all schools in Belgium."

While making these suggestions, children's values were implicitly expressed. Some of the children's opinions notably changed during the discussions with their peers and the researchers. When we finally proposed to use the money, if we would make any money at all, for additional research on the topic they simultaneously yelled "Yes!". In other words, an overall consensus was reached. Instead of being passive listeners, the children behaved as active participants from the very start. Due to this process, feelings of problem ownership emerged and children gradually uncovered and identified their personal values. This is considered to be an important step for building mutual trust between adults and child design partners. These values were documented by writing down children's reactions and by making a report immediately afterwards.

\section{REFLECTIONS AND CONCLUSION}

Complementing CHECk with interactive storytelling has been shown to be an effective way as to 'how' to inform participation and achieve ethical symmetry, but with it came new challenges. The subtle paradox of using a half lie (i.e., a made up story) to strengthen ethics and transparency in participatory design with children and the question of how to control the risk of possible influences from researchers on children's answer for final consensus should both be topics for further research.

Besides these challenges, interactive storytelling enabled us to create a shared narrative space. The shared narrative became the leitmotif, structuring the design activities and providing a clear end-goal for our child design partners. Since the story was fueled by children's input, it became tailored to their life-world, creating feelings of problem ownership and mutual trust.

By constantly probing children to think about and discuss their underlying motives (e.g. a group discussion about ownership of ideas, why questions embedded in drawing assignments, etc.), children's values emerged and developed recursively. Although CHECk was intended as an ethical probe to be used prior to and at the start of design activities, it became a vehicle for eliciting and negotiating values throughout the project.

\section{ACKNOWLEDGMENTS}

This study is part of (1) the EMSOC project (Emowerment in a Social Media Culture), funded by IWT (Angecy for Innovation by Science and Technology) and (2) a PhD research funded by the MAD-faculty (UHasselt).

\section{REFERENCES}

[1] Bekker, M., Beusmans, J., Keyson, D. \& Lloyd, P. (2003). KidReporter: a user requirements gathering technique for de- signing with children. Interacting with Computers, 15(2), 187-202.

[2] Bødker, S. (2006). When 2nd wave HCI meets 3rd wave challenges. In Proc. of the 4th Nordic conf. on HCI: changing roles (NordiCHI '06), ACM, NY

[3] Borning, A. \& Muller, M. (2012). Next Steps for Value Sensitive Design. CHI'12 Proceedings of the Conference on Human Factors in Computing Systems. ACM, NY.

[4] Buckingham, D. (2009). 'Creative' visual methods in media research: possibilities, problems and proposals. Media, Culture and Society, 31, 633-652.

[5] Christensen, P. \& Prout, A. (2002). Working with ethical symmetry in social research with children. Childhood, 9(4), 477 - 497.

[6] Dindler, C., Eriksson, E., Iversen, O.S., Lykke-Olesen, A. \& Ludvigsen, M. (2005). Mission from Mars: a method for exploring user requirements for children in a narrative space. Proceedings of the 2005 conference on Interaction design \& children (IDC '05). ACM, NY, 40-47.

[7] Friedman, B., Peter Kahn, J., \& Borning, A. (2006). Value sensitive design and information systems. In P. Zhang \& D. Galletta (Eds.), Human-Computer Interaction in Management Information Systems: Foundations. M.E. Sharpe, NY.

[8] Hallnäs, L., \& Redström, J. (2002). From use to presence: On the expressions \& aesthetics of every-day computational things. ACM Transactions on Computer-Human Interaction, 9(2), 106-124.

[9] Halloran, J., Hornecker, E., \& Stringer, M. (2009). The value of values: Resourcing co-design of ubiquitous computing. Co-Design, 4(5), 245-273.

[10] Iversen, O.S., Halskov, K., Leong, T.W. (2010). Rekindling Values in Participatory Design. PDC'10 Proceedings of the 11th Biennial Participatory Design Conference. ACM, NY.

[11] Löwgren. J., \& Stolterman, E. (2004) Thoughtful Interaction Design. MIT Press, Cambridge.

[12] Manders-Huits, N. (2011). What Values in Design? The Challenge of Incorporating Moral Values into Design. Science and Engeneering Ethics, 17(20), 271-287.

[13] Read, J., Horton, M., Sim, G., Gregory, P., Fitton, D., Cassidy, B. (2013). CHECk: A Tool to Inform and Encourage Ethical Practice in Participatory Design with Children. CHI '13 Extended Abstracts on Human Factors in Computing Systems. ACM, NY.

[14] Rokeach, M. (1973). The Nature of Human Values. Free Press, NY.

[15] Sengers, P., Boehner, K., David, S. and Kaye J. (2005). Reflective design. Proceedings of the 4th decennial conference on Critical computing: between sense and sensibility. ACM, NY.

[16] Sleeswijk Visser, F., Stappers, P.J., van der Lugt, R., Sanders, E.B.N. (2005). Contextmapping: Experiences from practice. CoDesign: International Journal of CoCreation in Design and Arts, 1(2), 119-149.

[17] Vines, J., Clarke, R., Wright, P., McCarthy, J., Oliver, P. (2013). Configuring participation: on how we involve people in design. Proceedings of the SIGCHI Conference on Human Factors in Computing Systems, 429-438. ACM, NY. 